Muséologies

Les cahiers d'études supérieures

muséologies

\title{
Les actions pédagogiques et culturelles au Département des Galeries du Muséum national d'histoire naturelle
}

\author{
Fabienne Noé, Gaud Morel et Johanne Landry
}

Volume 3, numéro 2, printemps 2009

URI : https://id.erudit.org/iderudit/1033573ar

DOI : https://doi.org/10.7202/1033573ar

Aller au sommaire du numéro

Éditeur(s)

Association Québécoise de Promotion des Recherches Étudiantes en

Muséologie (AQPREM)

ISSN

1718-5181 (imprimé)

1929-7815 (numérique)

Découvrir la revue

Citer cet article

Noé, F., Morel, G. \& Landry, J. (2009). Les actions pédagogiques et culturelles au Département des Galeries du Muséum national d'histoire naturelle.

Muséologies, 3(2), 192-201. https://doi.org/10.7202/1033573ar d'utilisation que vous pouvez consulter en ligne. 
Article quatorze

\section{Les actions pédagogiques et culturelles au}

\section{Département des Galeries du Muséum national}

\section{d'histoire naturelle}

FABIENNE NOÉ EST CHARGÉE DEPUIS PLUS DE DIX ANS DE LAACCUEIL DE LA PETITE ENFANCE À LA GRANDE GALERIE DE L'ÉVOLUTION. ELLE A MIS EN PLACE DES ATELIERS ET DES ACTIVITÉS PÉDAGOGIQUES ADAPTES AU JEUNE PUBLIC AINSI QUE DES DOCUMENTS DAIDE A LA VISITE POUR LES ENSEIGNANTS. ELLE COORDONNE DES PARTENARIATS AVEC DES CLASSES DE *ZONES DEEDUCATION PRIORITAIRES * AYANT POUR OBJECTIF DE FAIRE VENIR LES JEUNES AU MUSEE. ELLE PARTICIPE À DES FORMATIONS D'ENSEIGNANTS APRÈS UN PARCOURS PÉDAGOGIOUE DANS LES JARDINS BOTANIQUES, GAUOMOREL COORDONNE LES ACTIVITÉS PÉDAGOGIQUES DES GALERIES DU MUSÉUM, FANT POURLES SCOLAIRES QUE POUR LEPUBLIC FAMILIAL: ORGANISATION DE VISITES GUIDÉES ET ATELIERS SCIENTIFIQUES, CONCEPTION DE DOCUMENTS D'AIDE A LA VISITE, FORMATIONS D'ENSEIGNANTS, PROJETS SPÉCIFIQUES. BIOLOGISTE DE FORMATION, JOHANNE LANDRY A TRAVAILLÉ PENDANT PLUS DE 20 ANS DANS PLUSIEURS MUSÉES SCIENTIFIQUES, QUE CE SOIT EN CALIFORNIE, EN FRANCE, AU CANADA OU EN THAILANDE, APRĖS AVOIR DIRIGÉ LINSECTARIUM DE MONTRÉAI. PENDANT SEPT ANS, ELLE EST, DEPUIS OCTOBRE 2007, DIRECTRICE DU DEPARTEMENT DES GALERIES DU MUSÉUM NATIONAL DHISTOIRE NATURELLE A PARIS. ELLE SEST ENGAGÉE ACTVEMENT DANS PLUSIEURS ASSOCIATIONS MUSÉALES ET A RÉDIGÉ DE NOMBREUSES PUBLICATIONS DANS LE DOMAINE DE LA MUSÉOLOGIE ET DU DÉVELOPPEMENT DURABLE. [landry@mnnnfr] 


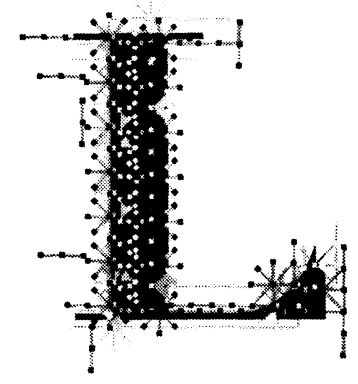

L'histoire du Jardin des plantes débute en 1635 avec la création du Jardin royal des plantes médicinales. Déjà, à cette époque, des conférences publiques (anatomie, botanique, chimie...) sont offertes gratuitement afin de former les apothicaires et les médecins. Le Muséum national d'histoire naturelle est créé par un décret en 1793, au moment de la chute de la royauté et du début de la Convention. L'histoire du Jardin des plantes et du Muséum est bien documentée et a fait l'objet de nombreux ouvrages, tant scientifiques que de vulgarisation. Le Muséum national d'histoire naturelle de Paris possède plusieurs sites : le Musée de l'Homme au Trocadéro, le zoo de Vincennes dans l'est de Paris et le Jardin des plantes dans le $5^{\mathrm{e}}$ Arrondissement où se trouvent la ménagerie et les galeries (Géologie et Minéralogie, Paléontologie et Anatomie comparée, Évolution). De plus, le Muséum gère 14 sites régionaux qui lui permettent d'étudier les divers écosystèmes locaux dont les stations marines de Dinard et de Concarneau et le Jardin botanique de Menton ${ }^{[1]}$.

Cet article, qui fait suite à une conférence donnée par Fabienne Noé aux étudiants de l'École du Louvre en septembre 2008. Il décrit uniquement les activités pédagogiques et culturelles du Département des Galeries qui comprend trois galeries d'exposition ouvertes au public.

La Grande Galerie de l'Évolution (GGE) (ancienne Galerie de Zoologie réalisée en 1889 par l'architecte Louis-Jules André) fut rénovée en 1994 par les architectes Paul Chemetov et Borja Huidobro qui ont brillamment conservé l'esprit du lieu. Cette galerie présente des animaux actuels selon le thème de la biodiversité comme résultat de l'évolution de la vie. La scénographie (réalisée par René Allio) a pour but de mettre en valeur la beauté des spécimens naturalisés et de déclencher l'émotion chez les visiteurs. Une thématique autour des relations 
Consulter le site du Muséum: $<$ http://www.mnhn.fr/ museum/foffice/tous/tous/ GuideDecouverte/> entre l'homme et la nature introduit la notion de développement durable et fait le constat que des espèces disparaissent régulièrement de la planète.

La Galerie de Paléontologie et d'Anatomie Comparée (GPAC), réalisée par l'architecte Frédéric Dutert en 1898, est restée dans son état original de la fin du XIXe siècle. Sous la direction scientifique du professeur et évolutionniste Albert Gaudry, le concept de la Galerie de Paléontologie ( $2^{e}$ étage) présente les animaux suivant leur apparition au cours des grandes époques géologiques. Elle expose toujours les mêmes fossiles et des moulages de squelettes d'animaux très spectaculaires, comme les dinosaures. Malgré son aspect ancien, elle fascine les visiteurs qui viennent nombreux, souvent avec des enfants. La Galerie d'Anatomie Comparée ( $\mathrm{r}^{\mathrm{e}}$ étage) présente des squelettes d'animaux actuels classés par grands groupes. On y trouve certains spécimens ayant vécu à la ménagerie du Roy aux XVII et XVIII siècles ainsi que d'autres provenant d'animaux naturalisés qui sont présentés à la Grande Galerie de l'Évolution. Cette galerie, très spectaculaire par la richesse et la diversité de ses collections historiques, est très populaire auprès des étudiants en art qui viennenty dessiner régulièrement. Le Muséum doit rénover prochainement cette galerie (éclairage, muséographie, services à la clientèle...), tout en conservant l'aspect historique unique des deux salles principales. premier bâtiment construit pour devenir un musée. Elle présente actuellement deux petites salles d'exposition : la salle des Cristaux géants et la salle du Trésor, où les visiteurs peuvent admirer deux collections spectaculaires de minéraux. Au-delà de la beauté des spécimens exposés, le propos scientifique est difficile à illustrer et le public y vient moins nombreux que dans les deux autres galeries ouvertes au public. La grande nef est parfois utilisée pour présenter des expositions temporaires fort recherchées, telles que Diamants. L'exposition actuelle, qui nous vient du Musée de la civilisation à Québec, propose le thème de L'Or des Amériques. Nous préparons un projet de rénovation majeur pour cette galerie d'ici les prochaines années.

Les collections présentées dans ces trois galeries ont été récoltées depuis 200 ans par des naturalistes voyageurs. Elles sont actuellement le support de nombreux travaux scientifiques menés par les 500 chercheurs du Muséum, ce qui fait du Muséum national d'histoire naturelle l'un des principaux muséums dans le monde (avec celui de Londres et de Washington), que ce soit par l'étendue de ses collections ou en raison du personnel scientifique qui y travaille. 


\section{Les publics}

La fréquentation des trois galeries du Muséum national d'histoire naturelle est importante puisqu'elle frôle le million de visiteurs par année, en fonction des expositions temporaires qui y sont présentées. Le public comprend deux catégories principales, le public individuel et les groupes scolaires. Le public individuel est constitué de familles avec de jeunes enfants, notamment à la Grande Galerie de l'Évolution. Ce public, fortement éduqué, se concentre principalement les samedis et les dimanches et pendant les semaines de congés scolaires (octobre, décembre, février et avril).

TABLEAU 1

en 2008

$\begin{array}{ll}\text { GALERIE } & \text { NOMBRE DE VISITEURS/ANNÉE } \\ \text { Grande Galerie de l'Évolution } & 689163 \\ \begin{array}{l}\text { Galerie de Paléontologie } \\ \text { et d'Anatomie comparée }\end{array} & 280441 \\ \begin{array}{l}\text { Galerie de Géologie } \\ \text { et de Minéralogie }\end{array} & 108382 \\ \end{array}$

Le public individuel représente $80 \%$ des visiteurs des galeries du Muséum. Les groupes scolaires représentent environ $20 \%$ et se répartissent ainsi : pour l'année 2007, 4900 classes en GGE, 1211 classes en GPAC et 126 classes en GMG, pour un total d'environ 156 ooo enfants. Les tranches d'âge sont :

- $27 \%$ cycles 1 et 2 (maternelle, CP, CE1), de 3 à 7 ans;

- $37 \%$ cycle 3 (CE2, CM1, CM2), de 8 à 11 ans;

- $21 \%$ collèges, de 12 à 15 ans;

- $14 \%$ lycées, de 16 à 18 ans.

D'autres types de groupes sont également accueillis : les centres de loisirs, les groupes d'adultes, les personnes en situation de handicap ainsi que les enseignants. 
Conception et programmation des activités pédagogiques

Diverses activités sont conçues par l'équipe pédagogique et programmées selon les types de publics ainsi que les périodes de l'année.

\section{Activités pour le public familial}

- Des visites guidées sont proposées le samedi dans les galeries permanentes et les expositions temporaires. Elles sont réalisées par des conférenciers du Muséum.

- Des ateliers sont offerts aux enfants ( 7 à 11 ans) pendant les vacances scolaires sur des thématiques diverses : Le tour du monde des coquillages, Le fil du temps, Les volcans, Les carnivores et les herbivores... Ces ateliers sont conçus comme un complément à la visite et peuvent s'adapter aux thèmes des expositions temporaires. Ils permettent aux enfants de manipuler les collections pédagogiques, ce qu'ils ne peuvent pas faire lors de la visite dans le musée. La manipulation de véritable matériel (fossiles, squelettes, animaux naturalisés) suscite la curiosité et incite les enfants à poser des questions.

- Il y a aussi des ateliers pour les très jeunes enfants (3 à 6 ans), où les collections sont mises en scène dans un espace théâtralisé et les thématiques sont abordées par le biais de la manipulation, de l'observation, du plaisir et du jeu: Du bout des doigts (atelier tactile dès 3 ans), Le voyage de Théodore Flocki, Cric crac croc montre-moi tes dents... Selon les thèmes d'exposition, certains ateliers font intervenir des artistes: Danser les animaux, L'atelier sonore du dragon, Fabriquer sa mouche...

- Des parcours jeux, distribués aux familles à l'accueil, sont des aides à la visite pour les expositions temporaires et permanentes. Ils sont conçus soit à l'interne soit dans le cadre de partenariat presse (par exemple l'exposition Incroyables cétacés !).

- Depuis quelques mois, une nouvelle expérience de médiation humaine est à l'essai : pendant certaines périodes de forte fréquentation, des animateurs interviennent dans la Grande Galerie de l'Évolution avec du matériel pédagogique dans le but d'attirer la curiosité des visiteurs et d'entrer en contact directement avec le public pour discuter d'enjeux environnementaux. 


\section{Activités pour les scolaires}

En tant que musée de l'éducation nationale, le Muséum a une mission éducative forte auprès des jeunes. Les enseignants viennent y chercher une illustration des programmes scolaires et sont attirés par la qualité des présentations et la notoriété des galeries dans le monde éducatif. Deux types de prestations leur sont proposés :

- Des visites guidées d'une durée variant de 60 à 90 minutes. Les thèmes sont nombreux et s'adaptent aux programmes scolaires. Les conférenciers sont des étudiants de haut niveau. Les enseignants de lycée viennent chercher la qualité du discours scientifique, basé sur l'observation des spécimens, et apprécient particulièrement la rencontre avec de jeunes chercheurs. Malheureusement, il est actuellement impossible de répondre à la forte demande des professeurs pour ce type de prestation.

- Des ateliers permettent la manipulation et s'adressent principalement aux jeunes enfants. Les objets de collection que les enfants peuvent s'approprier déclenchent l'émotion, l'intérêt et le questionnement. Ces séances se déroulent en petits groupes. Pour les plus jeunes, le mime est largement utilisé, par exemple dans l'atelier sur la locomotion. Un soin particulier est apporté à la conception de ces ateliers afin de valoriser la spécificité du Muséum : qualité du matériel utilisé, qualité du discours scientifique, spécialisation des médiateurs pour les plus jeunes...

\section{Les projets particuliers et les partenariats}

Le service pédagogique est régulièrement sollicité pour des projets "à la carte ", conçus avec les partenaires.

- L'accueil de classes d'enfants défavorisés (Réseau d'éducation prioritaire) - une formation est assurée aux enseignants, l'équipe pédagogique suit les projets et un bilan est fait en fin d'année. Elle permet aux enfants défavorisés d'avoir un accès privilégié au musée. Pour l'équipe pédagogique du musée c'est par ailleurs un moyen de cibler au mieux les attentes du corps enseignant et de tester de nouveaux thèmes et ateliers. 
Les classes inter-musées - une classe participe à des activités dans deux musées différents sur un même thème : le thème des animaux avec le Centre Pompidou ou le musée d'Orsay, les voyages scientifiques avec le musée de la Marine...

- Les classes Muséum - une classe rencontre un chercheur ou un technicien sur un sujet particulier (les dinosaures, les milieux polaires, la taxidermie...).

\section{Les formations pour les enseignants}

Ce sont des visites "galeries-mode d'emploi " qui permettent aux enseignants de se repérer dans l'espace et d'avoir une vue d'ensemble du lieu et des thématiques traitées: ce dispositif facilite la préparation pédagogique et la coordination logistique de leur visite avec les élèves. Ces formations sont le plus souvent demandées par les IUFM (instituts universitaires de formation des maîtres) dans le cadre de la formation continue ou initiale des enseignants. Elles sont proposées également à tous les enseignants les mercredis, sur inscription.

\section{Activités pour les publics en situation de handicap}

La Grande Galerie de l'Évolution et la Galerie de Paléontologie et d'Anatomie comparée ont reçu le label Tourisme et Handicap. Des activités adaptées à divers types de handicap y sont proposées, tant pour les groupes que pour le public individuel. Des partenariats sont menés avec des institutions pour un suivi d'activités au long de l'année scolaire.

- Pour les personnes avec une déficience visuelle, des visites tactiles dans les galeries permanentes et certaines expositions temporaires sont proposées (Dragon, Mammouth, Cétacés) et certains ateliers scolaires ont été adaptés. Le public est autorisé à toucher certains animaux en présence du conférencier et du matériel complémentaire est mis à sa disposition (maquettes, figurines...).

- Des visites et des ateliers en langue des signes française réalisés par des conférenciers sourds sont proposés aux personnes malentendantes. 
- Des conférenciers formés tout spécialement animent des ateliers et des visites pour les personnes ayant une déficience intellectuelle.

- La Grande Galerie de l'Évolution est accessible en fauteuil roulant : toutes les activités proposées au public sont donc accessibles aux visiteurs avec une déficience motrice.

\section{Accompagnement des expositions temporaires}

Des programmes d'activités culturelles sont mis en place en lien avec les expositions temporaires : séances de films et débats avec des spécialistes, cours publics et conférences par des chercheurs du Muséum...

\section{Conclusion}

Le Muséum national d'histoire naturelle propose, dans ses galeries, une programmation variée et ciblée pour les différents publics qui les fréquentent. Tout en conservant l'attrait de l'objet et l'émotion qu'il suscite, il est essentiel de maintenir une médiation humaine de qualité afin de transmettre un contenu scientifique rigoureux. Actuellement, de nouvelles approches d'animation ponctuelle sont développées pour favoriser l'interaction avec les publics et les sensibiliser à la perte de biodiversité que nous observons sur notre planète. 


\section{Summary}

[Translated by Micheline Giroux-Aubin]

The Muséum national d'histoire naturelle owns several sites in France (Dinard, Concarneau, Menton, Brunoy...), including three in the Paris area: the Musée de I'Homme, the Vincennes Zoo, and the Jardin des plantes in the 5th Arrondissement. The history of the Jardin des plantes goes back to the 17th century and is a Mecca for the history of sciences (botany, zoology, mineralogy, palaeontology...), with scientific researchers such as Buffon, Cuvier, Geoffroy St-Hilaire, Lamarck...

In addition to the botanical garden and the zoo, there are three galleries in the Jardin des Plantes: the Grande Galerie de l'Évolution (formerly the zoology gallery created in 1889 and renovated in 1994 by architects Chemetov and Huidobro), the Galerie de Paléontologie et d'Anatomie Comparée (1898), and the Galerie de Minéralogie et de Géologie (1837).

The collections presented in the three galleries have been collected by travelling naturalists over the last 200 years. They constitute the basis of numerous scientific works conducted by the Muséum's 500 researchers, making the Muséum national d'histoire naturelle one of the world major museums (with the London and the Washington museums), due to the extent of its collections and its scientific workforce. 
Visitors to the Muséum galleries come for the permanent and temporary expositions, mostly with their families $(80 \%)$, but also in groups $(20 \%)$. Various activities are organized to attract these audiences: guided tours, educative workshops, game itineraries, and special one-off animations in the galleries. Specific programs are also designed for school groups, either with researchers of the Museum (museum classes) or with associated museums (Orsay, Beaubourg...). Teacher trainings sessions are also offered in order to help them use our galleries independently.

With the help of an expert collaborator, a series of programs for the handicapped, be it a visual, hearing, intellectual, or motor disability have been created. The museum is in the process of setting up a gallery on the theme of biodiversity, dedicated to children, where museographic supports for that kind of public will be available.

The galleries of the Muséum national d'histoire naturelle propose varied programs, targeted at the different audiences who visit. While retaining the attraction of the object and the resulting emotion, we believe it is essential to also maintain a quality human mediation in order to transmit accurate scientific contents. New special interpretation approaches are currently developed to promote interaction with audiences and heighten their awareness related to the loss of biodiversity that is now occurring on our planet. 